\title{
REMOVAL OF SMALL-SIZED KIDNEY STONES FRAGMENTS USING INVERTED FLUSHING PROCEDURE IN URETEROSCOPY
}

\author{
Hradetzky D ${ }^{1}$, Strub K ${ }^{1}$, Sepassi $T^{1}$, Möltgen $T^{2}$ \\ ${ }^{1}$ Insitute for Medical and Analytical Technologies, University of Applied Sciences and Arts \\ Northwestern Switzerland, Switzerland \\ ${ }^{2}$ Kantonsspital Aarau AG, Aargau, Switzerland \\ david.hradetzky@fhnw.ch
}

\begin{abstract}
The minimal invasive removal of renal stones is restricted by their size. As currently no active removal of fragments smaller than $1 \mathrm{~mm}$ in diameter is available, we investigated on the change of flow direction of flushing media during ureteroscopy, to obtain an active removing mechanism through the working channel of the endoscope. Using a $3 D$ model of a kidney we measured the removal rate using this inverted flow and obtained a removal rate of up to $58 \%$ during 5 minutes inverted flushing of renal pelvis at natural kidney pressure values, while no removal was observed applying standard flushing procedure.
\end{abstract}

Keywords: lithotripsy, ureteroscopy, kidney stone removal, inverted flushing

\section{Introduction}

Ureteroscopy is a well-established minimal invasive procedure for the removal of kidney stones through the urinary tract. Therefore a ureteral access sheath is introduced up to the renal pelvis, through the urethra, bladder, and ureter in order to obtain a safe access for the ureteroscope to the renal pelvis.

Within this procedure smaller kidney stones are removed using the endoscopic access in combination with a mechanically assisting tool like Baskets or Stone extractors for capturing the stones. The removal of captured stones is conducted either through the working channel of the ureteroscope or by pulling out the whole endoscope, depending on the stone size. Larger stones may be broken in smaller fragments, using laser lithotripsy or extracorporeal shock wave lithotripsy. Removal of smaller fragments $(<1 \mathrm{~mm})$, occurring as unplanned secondary effect during fragmentation for example, is nowadays limited to the spontaneous release through natural urination. As remaining fragments increase the risk of a further stone event [1] a removal of all kidney stones including small sized fragments is preferred.

In this paper we investigate the effects of a reversed flushing method on the removal rate for small kidney stones fragments, using the working channel of the ureteroscope as an outlet for flushing media.

\section{Methods}

Our approach is based inverting the flow of flushing media, to obtain a focused outflow through the working channel of an Olympus URF-P5 flexible ureteroscope. This configuration results in an increased flow velocity. To obtain this inverted flow we modified the proximal port of a Flexor ${ }^{\circledR}$ ureteral access sheath from Cook Medical, shown in Figure 1 , by adding a silicone check valve and additional sealing facilitating the flushing media to flow through the gap between ureteroscope $(\varnothing 3.1 \mathrm{~mm})$ and flex sheath $(\varnothing 4 \mathrm{~mm})$ towards the renal pelvis. The outflow of flushing media is therefore restricted to the working channel. As the working channel $(\varnothing 1.2 \mathrm{~mm})$ offers a roughly five times smaller cross section $\left(1.1 \mathrm{~mm}^{2}\right)$ compared to the gap area $\left(5.0 \mathrm{~mm}^{2}\right)$, the outflow velocity will be significantly higher than the inflow velocity, resulting in a sucking effect. This sucking action is expected to enhance the removal of small kidney stone fragments.

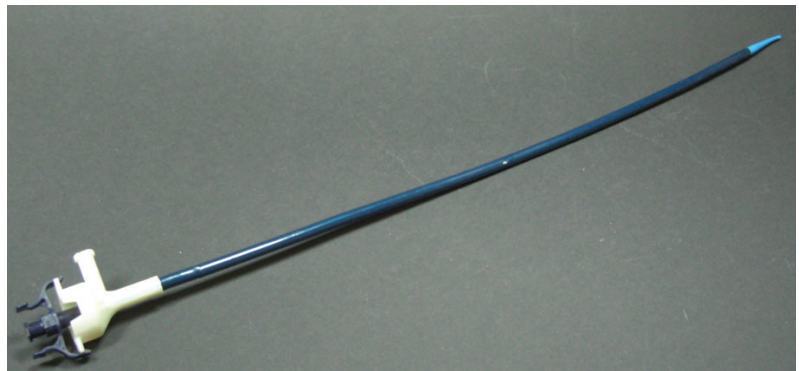

Figure 1: Modified Flexor ${ }^{\circledR}$ ureteral access sheath with additional check valve and sealing

In order to obtain a realistic experimental scenario we used CT data of a human renal pelvis to create a 3D model using additive manufacturing procedure [2] on an Eden250 ${ }^{\mathrm{TM}} 3 \mathrm{D}$ Printing System (Objet). To assure a visual and manual access to the cavity of the renal pelvis model, we used a sliced device covered with removable Plexiglas window on top and bottom, shown in Figure 2 left.
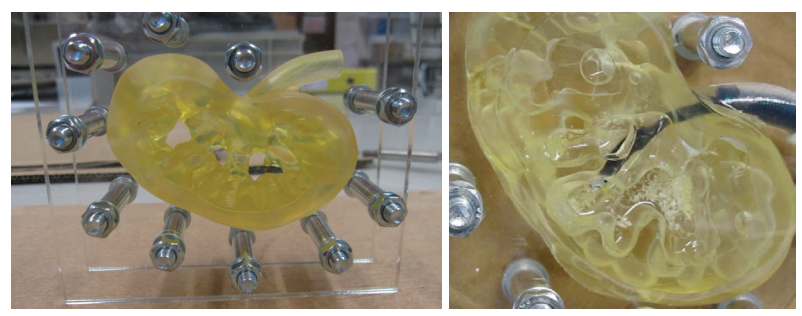

Figure 2: 3D model of the kidney for testing the inverted flow concept made of FullCure ${ }^{\circledR} 720$ (Objet) (left) and during the experiments with an inserted Flexor ${ }^{\circledR}$ ureteral access sheath and ureteroscope (right) 
The flushing media reservoir is adjustable in height, representing a clinical environment scenario and enabling different flow rates.

Struvit kidney stones from patients were used as object for removal. To obtain stone sizes in the small range (approx. $1.5 \mathrm{~mm}$ and below) we used a mortar for fragmentation.

For evaluation of the removal rate we introduced a wellknown amount of stone fragments into the kidney and proceeded with flushing experiments. During the flushing the endoscope was used by a medical unskilled person for duration of 5 minutes, intending to collect as much fragments as possible. The flushing media outflow, including $t$ the removed fragments was collected, dried $\left(60\right.$ min @ 100 $\left.{ }^{\circ} \mathrm{C}\right)$ and weighed. The removal rate was then determined as the weight ratio of removed fragments vs. initially introduced fragments. Applying the proposed drying procedure on $100 \mathrm{mg}$ fragments, a standard deviation of $1 \%$ was obtained. In order to obtain different flow rates, we used the reservoir at three different heights $(22,44,88 \mathrm{~cm})$. This heights lead to different pressures within the kidney, while the height of $22 \mathrm{~cm}$ is close to the natural pressure within the kidney $\left(15 \mathrm{~cm} \mathrm{H}_{2} \mathrm{O}\right)$ according to the Whitaker test [1].

For additional pressure measurement within the kidney we compared the pressure data (Freescale MPX 2010 differential pressure sensor) of our $3 \mathrm{D}$ renal pelvis model to a real pig kidney.

\section{Results}

In standard flow procedure, using working channel as inflow, we observed at 22 and $44 \mathrm{~cm}$ only turbulent flow of the fragments within single renal calix. Increasing the flow rate due to rising the reservoir height up to $88 \mathrm{~cm}$, a transfer of the fragments of one renal calix to the next and vice versa was observed. In all cases none of the fragments was removed through the outflow of flushing media.

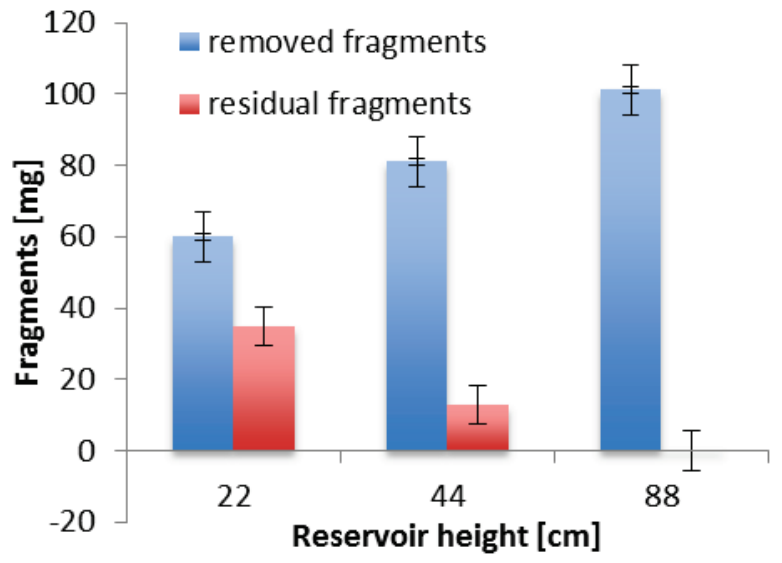

Figure 3: Mean value and standard deviation of removed and residual fragments after applying an inverted flow procedure for 5 minutes, based on 5 time repetition of the experiment.

Applying the inverted flow, using the working channel as outflow, a removal rate of $58 \%$ at $22 \mathrm{~cm}$ was achieved, increasing with the reservoir height as displayed in Figure 3. A complete removal of the introduced fragments at $88 \mathrm{~cm}$ within 5 minutes was obtained.

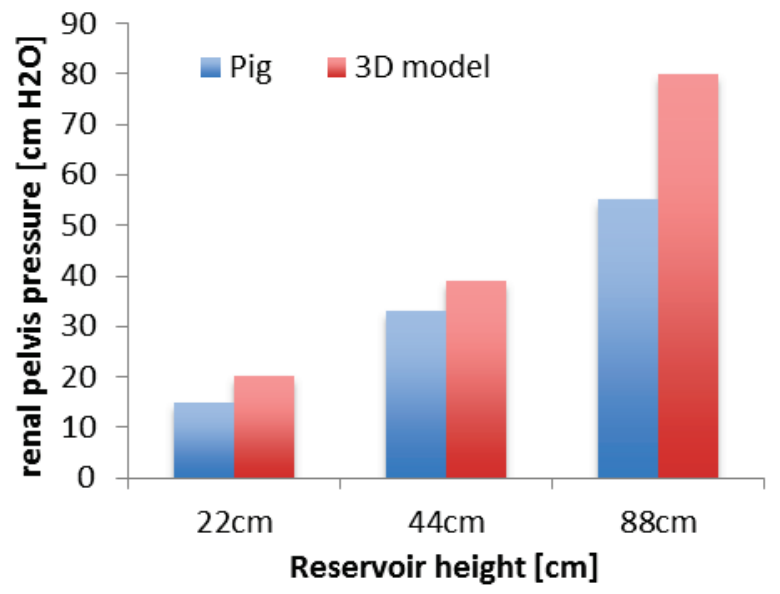

Figure 4: Comparison of the pressure data within a pig kidney and our 3D model during inverted flow procedure.

Additionally we investigated the pressure during the procedure within our 3D renal pelvis and compared the data obtained in an identical configuration using a renal pelvis of a pig. A reservoir height of $22 \mathrm{~cm}$ leads to a pressure of $18 \mathrm{~cm}$ $\mathrm{H}_{2} \mathrm{O}$ in our $3 \mathrm{D}$ model, while the pressure within the pig kidney is even more decreased down to $15 \mathrm{~cm} \mathrm{H}_{2} \mathrm{O}$. The obtained results are shown in Figure 4.

\section{Discussion}

Due to fact of inverting the fluidic flow an increasing of pressure obtained in the kidney was expected and observed, while almost the whole pressure drop occurs at the fluidic resistance of the working channel. But even when reducing the reservoir height, resulting in a decreased flow rate compared to standard flow procedure, a removal of kidney stone fragments was observed superior to no removal within the standard procedure. A complete removal of kidney stone fragments smaller than $1.5 \mathrm{~mm}$ within $5 \mathrm{~min}$. (@88 cm) in a model kidney was realized. Even at kidney pressures comparable to natural kidney pressure and therefore more suitable for clinical application (@22 cm), a removal rate of 58\% was obtained, providing a fragment removal superior to standard flow procedures.

Increasing the removal time, we expect that the complete removal of fragments should be possible even at low kidney pressures, decreasing the risk of gathering a new stone event, reducing the patients stress as well as overall treatment expenditures.

\section{Acknowledgment}

We would like to thank Olympus Schweiz AG for supporting us with a URF-P5 flexible ureteroscope.

\section{Bibliography}

[1] Baert AL, Fotter R, Knauth M, Sartor K. Pediatric Uroradiology. Berlin, Heidelberg: Springer Berlin Heidelberg, 2008.

[2] Gibson I, Rosen DW, Stucker B. Additive Manufacturing Technologies: Rapid Prototyping to Direct Digital Manufacturing: Springer US, 2009. 